\title{
Case - Bladder paraganglioma in a pediatric patient
}

\author{
A.K. Malhotra ${ }^{1}$; R. Yan ${ }^{1}$; R. Tabeshi ${ }^{2}$; H. Nadel ${ }^{3}$; H. Tran ${ }^{1}$; J. Masterson ${ }^{2}$ \\ ${ }^{1}$ Faculty of Medicine, University of British Columbia; ${ }^{2}$ British Columbia Children's Hospital, Department of \\ Urological Sciences; ${ }^{3}$ British Columbia Children’s Hospital, Department of Nuclear Medicine, Vancouver, BC, \\ Canada
}

Cite as: Can Urol Assoc J 2018 Feb. 6; Epub ahead of print. http://dx.doi.org/10.5489/cuaj.4937

Published online February 6, 2018

$* * *$

\section{Initial presentation}

A 12-year-old boy presented with a two-day history of gross hematuria and vomiting. Further questioning revealed a one-year history of position-induced paroxysmal headaches, polyuria, polydipsia, nocturia, dehydration, and lethargy. He denied any syncopal episodes, palpitations, night sweats, blurry vision, or anxiety attacks. He was noted to have normal pubertal development. An abdominal ultrasound was performed, and showed an 8.1 x $7.5 \mathrm{~cm}$ bladder mass on the posterior bladder wall with hypervascular components (Figure 1). The patient was brought to the operating theater for cystoscopy, which revealed a large, necrotic mass on the postero-lateral bladder wall. Bladder biopsy was performed. There were significant blood pressure fluctuations during the biopsy. Final pathology reported the mass to be a bladder paraganglioma (PGL).

The patient received a two-week course of alpha-adrenergic blockade with Prazosin and Amlodipine for blood pressure control prior to definitive surgery. Echocardiogram did not reveal significant cardiomyopathy. PET/CT scan showed no evidence of metastatic disease, but avid F18-fludeoxyglucose ([F-18]FDG) uptake was noted at the tumor site (Figure 2).

\section{Management}

The patient was brought back to operating theatre for treatment via partial cystectomy. The tumor was located near the right ureteric opening, and a ureteric stent was placed for 6 weeks. Pathology reported the resected PGL had negative surgical margins and no invasion into extravesicular fat. The patient was followed with urine metanephrine and blood pressure measurements every three months for surveillance. Subsequent medical genetics consultation revealed an underlying succinate dehydrogenase B (SDHB) gene mutation. First-degree patient relatives were subsequently investigated for the SDHB mutation. 


\section{Recurrence}

Three years post-resection, a routine endocrinological surveillance test detected elevated urine norepinephrine, normetanephrine and dopamine. An urgent FDG PET/CT scan revealed three avid soft tissue masses: a 5x9 mm right anterolateral lesion between the bladder wall and the inguinal canal, an enlarged 9x11mm right external iliac node, and a nonspecific density in the left paraspinal muscle at the level of the ninth rib. A two-week pre-operative course of alphaadrenergic blockade was administered in preparation for surgery. An intra-operative gamma probe procedure was utilized for lesion localization. Right sided avid common iliac nodes were sampled and PGL cells were histologically confirmed in one lymph node. Thirteen obturator nodes anterior to the bladder were resected and revealed benign histology with evidence of histiocytosis in five nodes. Paraspinal muscle biopsies were negative. Of the lesions visualized using PET/CT imaging, a single lymph node was positive for PGL cells. Endocrine consultation and bloodwork were negative for signs of disease post-resection. Further cytogenetic studies demonstrated a normal male karyotype 46XY with a balanced translocation t(5;19)(q31;p13). Continual surveillance is comprised of regular ultrasonography and urine catecholamine testing every three months with PET/CT scans only if either of these methods indicate abnormalities. Our center elected to perform regular cystoscopy for evidence of local recurrence or a new bladder primary. Currently, evidence around this protocol is lacking from literature.

\section{Discussion}

\section{Clinical presentation}

Pheochromocytomas (PCC) and PGLs are rare catecholamine-secreting tumours arising from either chromaffin tissue in the adrenal medulla or embryonic neural crest cells, respectively. ${ }^{1,2}$ PGLs may be found anywhere from the subocciput to the pelvic cavity. ${ }^{1}$ The incidence of these tumours is 2-8 per million, with most patients being diagnosed in the third to fourth decade. ${ }^{3}$ PCCs are associated with a lower rate of malignancy at less than $10 \%$, as opposed to PGLs, which have a malignancy rate up to $40 \%$. $^{2}$ Bladder PGLs are extremely rare, making up less than $0.05 \%$ of all bladder tumours, and less than $1 \%$ of all PCCs and PGLs. Malignancy rates specific to bladder PGLs are reported between $5-15 \%$, and are higher in hereditary conditions. ${ }^{4}$ It is therefore advisable to genetically screen patients presenting with malignant PCCs or PGLs for SDHx mutations. ${ }^{5}$ Zimmerman et al. was the first to report a case of PGL of the bladder in 1953, and since that time there have been fewer than 200 cases described. ${ }^{6,7}$ Bladder PGLs typically present with painless gross hematuria (50-60\%), hypertension (65-80\%), headaches, diaphoresis, and palpitations during micturition. ${ }^{8,9}$ Most of these tumors are hormonally active (83\%) and are commonly located in the submucosa either in the dome or the posterior wall near the trigone $(41 \%) .{ }^{9,10}$ 


\section{Familial paraganglioma syndromes}

Hereditary forms of PCC and PGL include von Hippel-Lindau disease, multiple endocrine neoplasia type 2, and type 1 neurofibromatosis. Associated predisposing genes include VHL, RET, NF1, SDHB, SDHC, SDHD, and SDHAF2, which may account for 40-45\% of PCCs and PGLs. ${ }^{3,11}$

Following a medical genetics consultation, our patient was found to have SDHB mutation. The succinate dehydrogenase enzyme is also known as Complex II in the mitochondrial electron transport chain, and consists of four subunits (A-D), with subunits B, C and D associated with PCC and PGL. SDHB-related tumor susceptibility (also known as PGL4) is inherited in an autosomal dominant manner. Compared to mutations in the other subunits, SDHB mutations are associated with a higher frequency of abdominal and pelvic PGLs, but lower frequency of head and neck PGLs. ${ }^{12}$

Mutations in the B subunit, a tumor suppressor gene, have been associated with more aggressive clinical outcomes, with high malignancy rates (34-71\%) ${ }^{13}$ and frequent occurrence of multifocal disease. ${ }^{12}$ Rates of penetrance vary between studies. Some reported $80-100 \%$ by age 70 , whereas other studies and reviews reported $45-52 \%$ by ages $35-60 ., 12,14,15$ However, not only is it important for first-degree relatives of affected individuals to undergo genetic testing, but also patients presenting with apparently sporadic PCCs and PGLs, especially when exhibiting qualities of PGL4 such as having malignant multifocal or extra-adrenal tumors, or early-onset presentation. Regular screening of at-risk patients and family members and early detection of disease reduces morbidity and complications. ${ }^{15}$

One proposed model relating SDHB mutations to PCC describes a pseudohypoxic state caused by impaired oxidative phosphorylation resulting from partial or complete loss of activity of the SDH enzyme ${ }^{14}$. It has been proposed that this induces the HIF-1 (hypoxia-inducible factor 1) pathway, which is known to cause the upregulation of genes involved in cellular metabolism, cell proliferation, apoptosis, glucose transport, and angiogenesis ${ }^{16}$.

\section{Investigations and imaging}

Pre-operative diagnostic tests include 24-hour urine or plasma fractionated catecholamines and metanephrines. However, these tests may be normal in certain tumours that only produce small levels of catecholamines or exclusively secrete dopamine. Therefore, measuring plasma dopamine or its metabolite may be useful. ${ }^{17}$ Chromogranin A is stored and released with catecholamines and can be measured in the plasma for identifying PCCs with a sensitivity of 8389\%. Chromogranin A may be useful for confirmatory testing in the setting of mildly elevated free plasma metanephrine levels, differentiating between benign and malignant disease, and gauging tumour response and relapse. ${ }^{1}$

There is a scarcity of evidence-based biochemical surveillance protocols for patients with hereditary PGL syndromes. This is due to the broad spectrum of genotypes and associated phenotypic patterns of tumour secretion products, location, and behavior. SDHB mutations are generally associated with large extra-adrenal tumours and high malignant potential. Furthermore, 
a predilection for dopamine and dopamine metabolite (methoxytyramine) secretion has been associated with SDHB gene mutations.X

Imaging studies for bladder paragangliomas include ultrasonography, computed tomography (CT), magnetic resonance imaging (MRI), and Molecular imaging scans using radiotracers. Bladder paragangliomas appear as hypoechoic, well defined lesions on ultrasonography; however, these findings are non-specific. ${ }^{9} \mathrm{CT}$ has a much higher sensitivity for extra-adrenal, metastatic, or recurrent tumours. Smaller lesions may appear homogenous on CT, while larger lesions appear heterogeneous due to hemorrhage and low-density necrotic areas. MRI is particularly useful in cases where ionizing radiation should be avoided, such as for children or pregnant women. PCCs are isointense to the liver, kidneys, and muscles on T1 imaging. On T2 imaging, the hypervascularity of the tumour makes it appear characteristically bright. MRI allows for visualization of vessel invasion and may be performed using contrast without risk of catecholamine release. Overall, the sensitivity of detecting extra-adrenal, metastatic, or recurrent PCC is 90\% versus 93-100\% for primary adrenal disease. ${ }^{18}$ The specificity associated with ultrasonography, CT and MR modalities is lower than that of molecular imaging.

Molecular imaging utilizes radiotracers to image human tissues; in combination with CT, this approach provides metabolic and functional detail layered onto anatomically accurate CT images. Depending on the nuclear properties of the radiotracers, either gamma radiation or positrons will be emitted as they accumulate in tissues, corresponding to SPECT/CT and PET/CT respectively. Relevant SPECT/CT scintigraphy methods for PCC and PGL imaging utilize spectrated radiopharmaceuticals including meta- ${ }^{123,131}$ I-benzylguanidine (mIBG) and somatostatin (SS) analogues labelled with ${ }^{111}$ In.

mIBG is a catecholamine analogue that shares both the uptake and storage mechanism with norepinephrine in the adrenal gland and lacks post-synaptic activity. ${ }^{10,11}$ Amino acid-based tracers such as ${ }^{111}$ In-pentetreotide is an SS analogue, which binds to SS receptors expressed by neuroendocrine tumours, and are therefore relevant to PGL and PCC imaging. ${ }^{19} \mathrm{mIBG}$ and ${ }^{111} \mathrm{In}$ pentetreotide are indicated for detection, staging, treatment $\left({ }^{131} \mathrm{I}\right)$ and monitoring therapy of PGL and PCC among other neuroendocrine tumours, though mIBG is considered the mainstay of PGL and PCC scintigraphy. ${ }^{20}$ The sensitivity and specificity of mIBG for PCC and PGL are reported to be $85-90 \%$ and $89-95 \%$, respectively. ${ }^{19,20} \mathrm{~m}^{-}{ }^{123}$ I-BG has a higher sensitivity than $\mathrm{m}^{-}{ }^{131}$-BG for imaging these tumours. ${ }^{21}$ Similar sensitivity and specificity is documented in malignant PCC or PGL using SS receptor analogue scintigraphy, though these values are based on smaller sample sizes. ${ }^{19}$ In the case of metastatic PCC, the sensitivity of ${ }^{111}$ In-pentetreotide scintigraphy has been reported to be higher than that achieved using mIBG. ${ }^{20}$ Importantly, the sensitivity of $\mathrm{m}-{ }^{123} \mathrm{I}-\mathrm{BG}$ for imaging PCC and PGL in familial SDHx mutation patients is $<50 \%{ }^{22}$

Positron Emission Tomography (PET/CT) is the primary investigative Molecular modality for PCC and PGL at our institution. There are several relevant radiolabelled isotopes; these include [F-18]FDG, which targets metabolically active cells as well as ${ }^{18} \mathrm{~F}$-fluoro-DOPA ([F-18]-DOPA), which interacts with catecholamine metabolism. ${ }^{22}$ [F-18]-DOPA is associated 
with a sensitivity and specificity of $91 \%$ and $95 \%$ respectively. ${ }^{23}$ Studies limited by small sample sizes suggest a role in staging and restaging for [F-18]-DOPA PET/CT in familial PGL syndromes with low malignant potential. ${ }^{24}$ As PGL cells become phenotypically less differentiated, the catecholamine metabolic function is hypothesized to become less regulated, reducing the utility of [F-18]-DOPA, but improving the utility of [F-18]FDG. ${ }^{24}$ Using [F18]FDG is is reported to be $90 \%$ sensitive in metastatic or highly malignant PGL, however, [F18]FDG accumulates in all rapidly dividing tissues and therefore remains non-specific for PCCs and PGLs. ${ }^{25}$ Other radiolabeled isotopes, such as [C-11]hydroxyephedrine, and [C-11]ephedrine are specific for paragangliomas, but the complex radiopharmaceutical development infrastructure required to feasibly produce these isotopes limits their utility in many centers including ours. ${ }^{18}$

There is growing evidence to suggest the utility of Gadolinium-68 (GA-68) labelled somatostatin analogue tracers using the PET/CT modality. One such example is GA-68 1,4,7,10tetraazacyclododecane-1,4,7,10-tetraacetic acid(0)-Tyr(3)-octreotate (GA-68-DOTATATE). Several somatostatin receptor isozymes have been demonstrated in PCC and PGL tissue and therefore these receptors make useful imaging targets. ${ }^{26,27}$ In a prospective study comprised of 17 SDHB mutation positive patients with metastatic PCCs or PGLs, GA-68-DOTATATE-based tracers demonstrated lesion-based detection rates of $98.6 \%$. This was higher than all other imaging modalities compared - including FDG-18. ${ }^{28}$ GA-68 tracers are not currently used at our institution.

We utilized an [F-18]FDG tracer to visualize the presenting and recurrent pathology. Due to the renal excretory pathway of [F-18]FDG, visualization of the bladder lesion was challenging. This issue has previously been reported in renal and bladder PGL cases. ${ }^{18}$ There was a discrepancy between tumour recurrence visualized on functional [F-18]FDG imaging and positive PGL biopsies taken from the three avid regions following dissection. Current literature has not yet elucidated a clear mechanism for these false positives on imaging. It was previously believed that since SDH-related PGLs, like VHL-related tumors, exhibited the Warburg effect, this explains the effectiveness of [F-18]FDG imaging for these tumors. However, evidence has since suggested that the increased glucose uptake in SDH paragangliomas is instead associated with increased expression of glucose and hexose transporters through the HIF-1 pathway rather than through metabolic changes. ${ }^{29}$ If this cellular phenotypic change takes place in the premalignant phase of cellular transformation, we suggest that this could provide a molecular basis for the false positive Molecular imaging.

\section{Operative management}

Preoperative goals include establishing adequate control of blood pressure and achieving euvolemia. Alpha-blockade for 7-14 days should be used to normalize blood pressure. For patients with cardiac arrhythmias or tachycardias, beta blockade can be used after alphablockade has been established. Volume expansion immediately prior to surgery is also recommended. ${ }^{29}$ 
Treatment options for bladder PCC include transurethral resection (TUR), radical cystectomy, and partial cystectomy. Das et al. performed a meta-analysis of 100 patients diagnosed with bladder pheochromocytomas and their treatment. 7\% of patients had TUR, 7\% had radical cystectomy, and $86 \%$ had partial cystectomy. The authors concluded that in the setting of localized disease, partial cystectomy may be satisfactory. However, extensive disease, positive nodal involvement or pelvic lymphadenopathy may indicate a radical procedure. ${ }^{7}$ The equivocal distinction between benign and malignant PCC on pathological examination may not always be definitive. Malignancy is typically proven with demonstration of chromaffin cells in non-chromaffin tissues. ${ }^{30}$

Postoperatively, the patient should be monitored to prevent possible cardiac and metabolic complications, such as hypoglycemia and hypotension. ${ }^{29}$ PGL and PCC recurrence is rare (4.6-6.5\%). However, follow-up is recommended. Harari et al suggest following SDHB gene positive patients or those with greater than 3 recurrence risk factors (elevated 24-hour urine dopamine, poor pathology characteristics, PASS $>6$ ) with plasma metanephrines every 6 months for 2 years, then annually for 10 years, and finally every 5 years for life. Otherwise, for all other patients, yearly plasma metanephrines for 5 years, then every 5 years for life is recommended. ${ }^{32}$

Patients with metastases can receive adjuvant chemotherapy, although reported outcomes are poor. ${ }^{7}$ Since these tumors arise from neural crest cell origin, a similar chemotherapy regimen to neuroblastoma is utilized, consisting of cyclophosphamide, vincristine, and dacarbazine (CVD). Huang et al evaluated 18 patients with metastatic PGL treated with CVD regimen. Patients included had good bone marrow, renal, and hepatic function with Karnofsky score > 30\%. Patients were followed with serial CT and MIBG imaging every 6-16 weeks. Partial or complete responders had a median survival of 3.8 years post chemotherapy, and all others had a median survival of 1.8 years. ${ }^{31,32}$

\section{Conclusion}

Bladder PGL is a rare catecholamine secreting neoplasm arising from chromaffin cells of the sympathetic nervous system within the bladder wall. Our patient presented with gross hematuria. Following appropriate diagnostic workup, he was treated with partial cystectomy. He has continued to have surveillance investigations and experienced one episode of biochemical recurrence during which PGL cells were detected in an inguinal lymph node. After undergoing cytogenetic analysis, our patient was found to have a hereditary mutation in the succinate dehydrogenase B gene. The long-term prognosis of this variant is associated with a high malignant potential and stringent surveillance is therefore warranted indefinitely. Due to the complex physiologic alterations associated with the disease, a multidisciplinary approach to management and treatment is important. 


\section{References}

1. Andersen KF, Altaf R, Krarup-Hansen A, et al. Malignant pheochromocytomas and paragangliomas - The importance of a multidisciplinary approach. Cancer Treat Rev 2011;37:111-9.

2. Vanderveen KA, Thompson SM, Callstrom MR, et al. Biopsy of pheochromocytomas and paragangliomas: Potential for disaster. Surgery 2009;146:1158-66.

3. Fishbein L, Nathanson KL. Pheochromocytoma and paraganglioma: Understanding the complexities of the genetic background. Cancer Genet 2012;205:1-11.

4. Bhalani SM, Casalino DD, Manvar AM. Paraganglioma of the bladder. J Urol 2011;186:27980 .

5. Lenders JWM, Duh Q-Y, Eisenhofer G, et al. Pheochromocytoma and paraganglioma: An Endocrine Society clinical practice guideline. J Clin Endocrinol Metab 2014;99:1915-42.

6. Zimmerman IJ, Biron RE, MacMahon HE. Pheochromocytoma of the urinary bladder. $N$ Engl J Med 1953;249:25-6.

7. Das S, Bulusu N V., Lowe P. Primary vesical pheochromocytoma. Urology 1983;21:20-5.

8. Piédrola G, López E, Rueda MD, et al. Malignant pheochromocytoma of the bladder: Current controversies. Eur Urol 1997;31:122-5.

9. Thrasher JB, Rajan RR, Perez LM, et al. Pheochromocytoma of urinary bladder:

Contemporary methods of diagnosis and treatment options. Urology 1993;41:435-9.

10. Baima C, Casetta G, Vella R, et al. Bladder pheochromocytoma: A 3-year follow-up after transurethral resection (TURB). Urol Int 2000;65:176-8.

11. Opocher G, Schiavi F. Genetics of pheochromocytomas and paragangliomas. Best Pract Res Clin Endocrinol Metab 2010;24:943-56.

12. Kantorovich V, King KS, Pacak K. SDH-related pheochromocytoma and paraganglioma. Best Pract Res Clin Endocrinol Metab 2010;24:415-24.

13. Timmers HJLM, Kozupa A, Eisenhofer G, et al. Clinical presentations, biochemical phenotypes, and genotype-phenotype correlations in patients with succinate dehydrogenase subunit B -associated pheochromocytomas and paragangliomas. J Clin Endocrinol Metab 2007;92:779-86.

14. Gottlieb E, Tomlinson IPM. Mitochondrial tumour suppressors: A genetic and biochemical update. Nat Rev Cancer 2005;5:857-66.

15. Favier J, Brière J-J, Strompf L, et al. Hereditary paraganglioma/ pheochromocytoma and inherited succinate dehydrogenase deficiency. Horm Res 2005;63:171-9.

16. Semenza GL. HIF-1 and human disease: One highly involved factor. Genes Dev 2000;14:1983-91.

17. Eisenhofer G, Siegert G, Kotzerke J, et al. Current progress and future challenges in the biochemical diagnosis and treatment of pheochromocytomas and paragangliomas. Horm Metab Res 2008;40:329-37.

18. Ilias I, Pacak K. Current Approaches and Recommended Algorithm for the diagnostic 
localization of pheochromocytoma. J Clin Endocrinol Metab 2004;89:479-91.

19. Pepe G, Bombardieri E, Lorenzoni A, et al. Single-photon emission computed tomography tracers in the diagnostics of neuroendocrine tumors. PET Clin 2014;9:11-26.

20. Ilias I, Chen CC, Carrasquillo JA, et al. Comparison of 6-18F-fluorodopamine PET with 123I-metaiodobenzylguanidine and 111in-pentetreotide scintigraphy in localization of nonmetastatic and metastatic pheochromocytoma. J Nucl Med 2008;49:1613-9.

21. Lenders JWM, Eisenhofer G, Mannelli M, et al. Phaeochromocytoma. Lancet 2005;366:66575.

22. Angelousi A, Kassi E, Zografos G, et al. Metastatic pheochromocytoma and paraganglioma. Eur J Clin Investig 2015;45:986-97.

23. Timmers HJLM, Kozupa A, Chen CC, et al. Superiority of fluorodeoxyglucose positron emission tomography to other functional imaging techniques in the evaluation of metastatic SDHB-associated pheochromocytoma and paraganglioma. J Clin Oncol 2007;25:2262-9.

24. Marzola MC, Chondrogiannis S, Colletti PM, et al. Succinate dehydrogenase mutationrelated paragangliomas: conventional versus PET/CT diagnostic work-up. Nucl Med Commun 2015;36:657-65.

25. Timmers HJLM, Chen CC, Carrasquillo JA, et al. Comparison of 18F-fluoro-L-DOPA, 18Ffluoro- deoxyglucose, and18F-fluorodopamine PET and 123I-MIBG scintigraphy in the localization of pheochromocytoma and paraganglioma. J Clin Endocrinol Metab 2009;94:4757-67.

26. Sharma P, Singh H, Bal C, et al. PET/CT imaging of neuroendocrine tumors with (68)Gallium-labeled somatostatin analogues: An overview and single institutional experience from India. Indian J Nucl Med 2014;29:2-12.

27. Ueberberg B, Tourne H, Redman A, et al. Differential expression of the human somatostatin receptor subtypes SST1 to SST5 in various adrenal tumors and normal adrenal gland. Horm Metab Res 2005;37:722-8.

28. Janssen I, Blanchet EM, Adams K, et al. Superiority of [Ga]-DOTATATE PET/CT to other functional imaging modalities in the localization of SDHB-associated metastatic pheochromocytoma and paraganglioma. Clin Cancer Res 2015;21:3888-95.

29. Mannelli M. Management and treatment of pheochromocytomas and paragangliomas. Ann $N$ Y Acad Sci 2006;1073:405-16.

30. Jain T, Basher R, Gupta N, et al. Unusual presentation of bladder paraganglioma: Comparison of ${ }^{131}$ I MIBG SPECT/CT and ${ }^{68}$ Ga DOTANOC PET/CT. World J Nucl Med 2015;15:65-7.

31. Huang H, Abraham J, Hung E, et al. Treatment of malignant pheochromocytoma/paraganglioma with cyclophosphamide, vincristine, and dacarbazine. Cancer 2008;113:2020-8.

32. Harari A, Inabnet WB. Malignant pheochromocytoma: A review. Am J Surg 2011;201:693701. 


\section{Figures and Tables}

Fig. 1. Transverse ultrasound image through the bladder identifies a large lobulated solid mass in the base of the bladder.

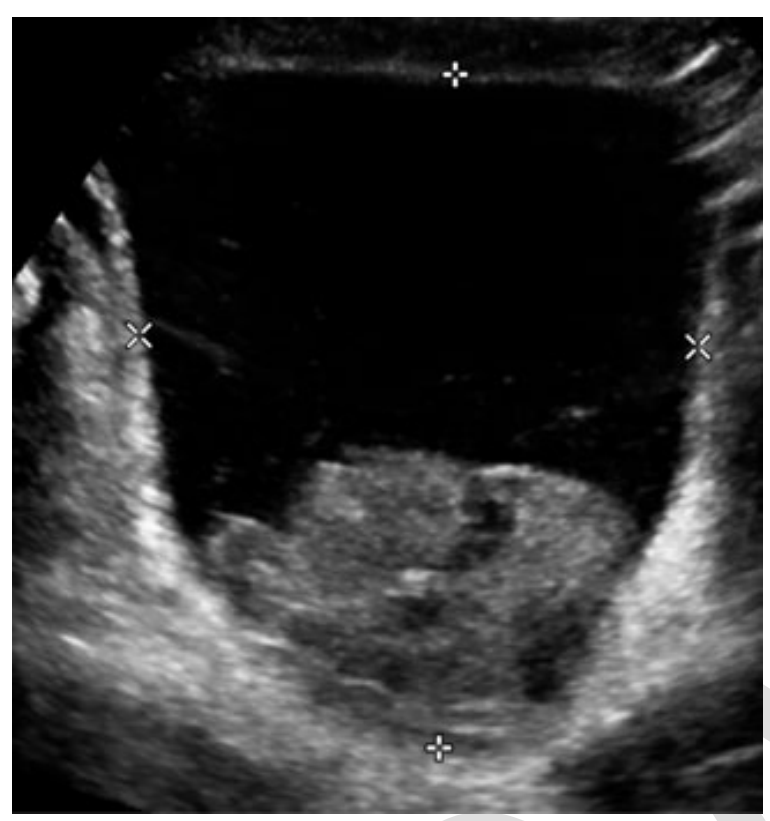

Fig. 2. 18- FDG PET/CT image in axial and coronal projections show increased FDG activity in the bladder mass (red chevron) that is indenting the bladder (black arrow). First row is CT, second PET and third fusion of PET/CT.
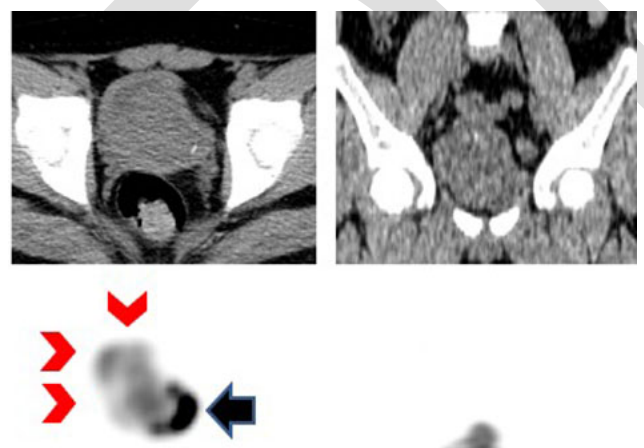

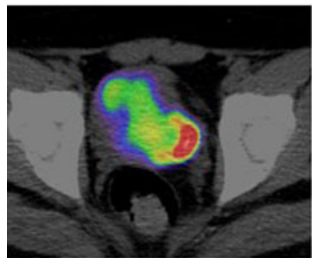

Axial

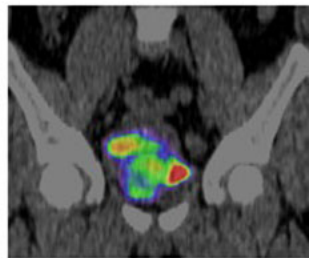

Coronal 